\title{
Trombosis bilateral del seno cavernoso en un paciente con enfermedad periodontal y diabetes mellitus. descripción de un
}

\section{caso}

\section{Bilateral cavernosus sinus thrombosis in a patient with periodontal disease and diabetes mellitus. description of a case}

\author{
Marcela Patiño-Arboleda (D', Juan Felipe Muñoz (D)!, José Mauricio Ocampo (iD³, María Teresa Calzada (D)+, José Mauricio \\ Cárdenas-Prieto iD;
}

1. Universidad Libre. Cali, Colombia. Correo: marcelapatino3091@gmail.com - https://orcid.org/0000-0002-8420-6334

2. Universidad Libre. Cali, Colombia. Correo: felipepipe26@gmail.com - https://orcid.org/0000-0002-1622-8873

3. Universidad Libre. Universidad del Valle. Cali, Colombia. Correo: jose.m.ocampo@correounivalle.edu.co - https://orcid.org/0000-0001-60844764

4. Universidad del Valle, Cali, Colombia. Correo: maria.t.calzada@correounivalle.edu.co - https://orcid.org/0000-0002-6534-909X

5. Universidad Libre. Cali, Colombia. Correo: josemaoneuro@gmail.com - https://orcid.org/0000-0003-4215-8378

Tipología: Reporte de caso clínico

Para citar este artículo: Patiño-Arboleda M, Muñoz JF, Ocampo JM, Calzada MT, Cárdenas-Prieto JM. Trombosis bilateral del seno cavernoso en un paciente con enfermedad periodontal y diabetes mellitus. descripción de un caso. Duazary. 2021 julio; 18(3): 131-140. Doi: https://doi.org/10.21676/2389783X.4239

\section{RESUMEN}

clave:

trombosis de la

vena; seno

diabetes

mellitus;

enfermedades

periodontales.
Palabras

cavernoso;

La trombosis de los senos cavernosos (TSC) corresponde a una entidad vascular poco usual, asociada con alta morbimortalidad y secuelas neurológicas. La etiología más frecuente son los procesos infecciosos a nivel de cabeza y cuello, que sirven como puerta de entrada para diferentes microorganismos, generando una respuesta inflamatoria que desencadena el evento trombótico. La enfermedad periodontal caracterizada por inflamación gingival, pérdida de tejido conectivo circundante y eventual del diente está asociada con el desarrollo de enfermedades sistémicas secundarias a los patógenos periodontales y mediadores inflamatorios producidos en la cavidad oral. Estos pueden diseminarse y producir complicaciones por contigüidad como eventos trombóticos a nivel del sistema nervioso central. A continuación, se presenta el caso clínico de un paciente que desarrolló una TSC bilateral después de ser sometido a un procedimiento periodontal.

\section{ABSTRACT}

Keywords: Thrombosis of the vein; Cavernous sinus; Diabetes mellitus; Periodontal diseases.
Cavernous sinus thrombosis is a vascular disease with an unusual presentation, associated with high morbidity, mortality and neurological sequelae. The most frequent etiological cause are the infectious processes at the head and neck level, being the gateway for different microorganisms that generate an inflammatory response that triggers the thrombotic event. Periodontal disease characterized by gingival inflammation, loss of surrounding connective tissue and eventual exfoliation of the tooth, is associated with the development of systemic diseases due to periodontal pathogens and inflammatory mediators that spread and produce complications due to contiguity such asthrombotic events in the central nervous system. We present the clinical case of a patient who developed a bilateral cavernous sinus thrombosis after undergoing a periodontal procedure. 


\section{INTRODUCCIÓN}

La trombosis venosa cerebral (TVC) constituye una enfermedad poco común asociada con alta morbimortalidad y complicaciones neuroftalmológicas ${ }^{1}$.Los primeros casos de TVC fueron reportados a principios del siglo XIX por Ribes y Abercrombie, y el primer caso de trombosis de los senos cavernosos (TSC) se describió en 1778 , siendo esta la localización menos común ${ }^{2,3}$.

La TSC representa una entidad grave asociada con un desenlace potencialmente fatal, con una mortalidad cercana al 30\%. Por lo tanto, el reconocimiento temprano y el inicio oportuno del manejo médico se asocian con resultados favorables ${ }^{4,5}$. Dentro de los factores asociados con su aparición se encuentran los de origen infeccioso secundarios a la invasión de microorganismos patógenos a nivel de cabeza y cuello ${ }^{6}$.

La etiología infecciosa se ve favorecida por la ausencia de válvulas en las venas tributarias del seno cavernoso, que permite un flujo sanguíneo bidireccional y predispone a un proceso séptico trombótico a este nivel, derivado de una infección por contigüidad, especialmente en senos paranasales, cavidad oral, órbitas y oído ${ }^{7}$. La enfermedad periodontal se relaciona con el desarrollo de enfermedades sistémicas secundarias a patógenos y mediadores inflamatorios producidos en la cavidad oral, y la diseminación de estos a sitios distantes o contiguos como el sistema nervioso central, proceso que se ve favorecido en individuos con algún grado de inmunosupresión ${ }^{8}$.

A continuación, se describe el caso clínico de un paciente adulto mayor con antecedente de diabetes mellitus no controlada e historia reciente de procedimiento periodontal en zona del cuadrante superior derecho, quien desarrolló trombosis séptica bilateral del seno cavernoso.

\section{REPORTE DE CASO CLÍNICO}

Paciente masculino de 68 años de edad, quien consultó al servicio de urgencias por un cuadro de cuatro días de evolución de náuseas, emesis de contenido alimentario en tres oportunidades, astenia y adinamia. Refirió como antecedentes patológicos hipertensión arterial y diabetes mellitus tipo 2 de 10 años de evolución y manejo farmacológico, con losartán (100 mg día) y metformina (850 $\mathrm{mg}$ dos veces al día), respectivamente. Además, se registró historia reciente de siete días previos a la consulta hospitalaria de un tratamiento periodontal en la zona del canino y primer premolar del cuadrante superior derecho (13 y 14 respectivamente).

Al ingreso se encontró con TA: 138/76 mmHg, FC: 90 Ipm, FR: $18 \mathrm{rpm}$ y $\mathrm{SatO}_{2}: 95 \%$ ambiente. En el examen físico se documentó leve eritema y edema en la encía de los dientes 13 y 14, asociado con higiene bucal deficiente. Los paraclínicos iniciales mostraron elevación en la PCR, leucocitosis y neutrofilia, glucosa en $429 \mathrm{mg} / \mathrm{dL}$, cuerpos cetónicos positivos en sangre, uroanálisis con presencia de glucosa más cuerpos cetónicos y gases arteriales con acidosis metabólica y anión GAP>15 $\mathrm{mEq} / \mathrm{L}$. Con estos hallazgos se consideró cetoacidosis diabética moderada por cumplir con los criterios diagnósticos tales como glucosa en plasma mayor de $250 \mathrm{mg} / \mathrm{dL}$, presencia de cuerpos cetónicos en sangre y orina, acidosis metabólica con elevación de anión GAP y, en este caso, ausencia de alteración del estado mental. Se ingresó a la unidad de cuidados intensivos para su respectivo manejo médico.

Tres días posteriores al ingreso hospitalario, el paciente inició con edema y eritema periorbitario derecho, asociado con pupila midriática no reactiva, reflejo corneano ausente y oftalmoplejía completa ipsilateral. El ojo izquierdo manifestó isocoria normorreactiva, movimientos oculares preservados y leve ptosis palpebral (figura 1). Con base en los hallazgos clínicos, se consideró que el paciente estaba cursando con síndrome del seno cavernoso, posiblemente de etiología infecciosa por el historial del procedimiento periodontal reciente, sumado con los hallazgos en la cavidad oral al examen físico de ingreso $y$, como factor de riesgo adicional, diabetes mellitus con pobre control metabólico por $\mathrm{HbA} 1 \mathrm{c}$ del $10 \%$ que lo predispone al desarrollo de procesos infecciosos. 


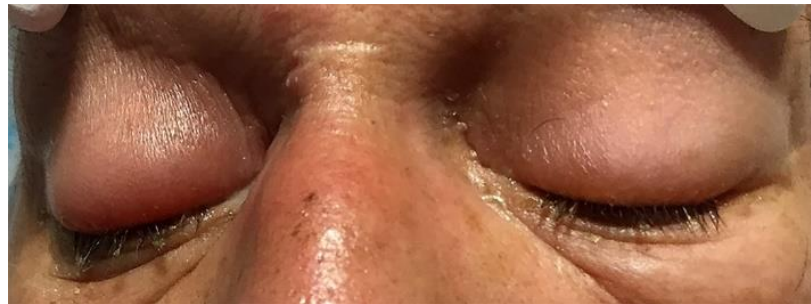

Figura 1. Edema y eritema a nivel periorbitario derecho.

Se inició anticoagulación con heparinas de bajo peso molecular más cubrimiento antibiótico con ceftriaxona, vancomicina y metronidazol. Se realizó resonancia magnética de silla turca simple $y$ contrastada con cortes finos, la cual mostró TSC derecho que explicaba las manifestaciones clínicas neurológicas, asociado con cambios inflamatorios en celdillas etmoidales y compromiso del antro maxilar derecho (figura 2). Se tomó adicionalmente TAC de senos paranasales en búsqueda de otros posibles focos de origen infeccioso contiguos a la cavidad oral periodontal, donde se obtuvieron hallazgos compatibles con una sinusitis etmoidomaxilar derecha, asociada con aumento en los volúmenes de tejidos blandos periorbitarios del mismo lado. Este proceso infeccioso posiblemente tuvo un origen odontogénico y se complicó con celulitis orbitaria ipsilateral.
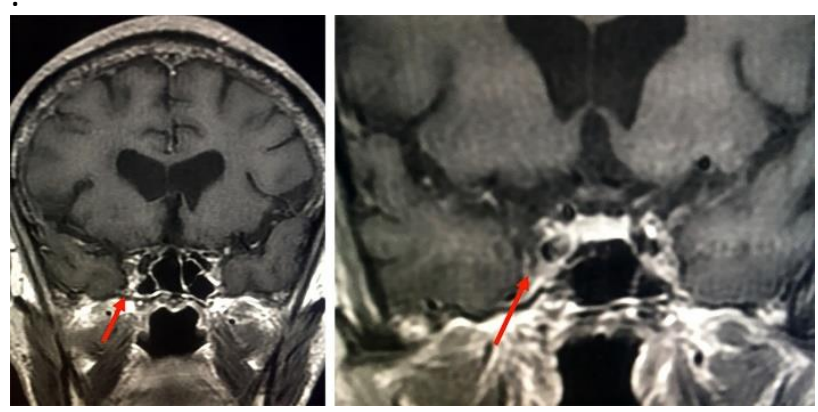

Figura 2. Resonancia magnética de silla turca donde se observa una trombosis del seno cavernoso derecho y abombamiento de sus márgenes laterales.

Durante la estancia en la unidad de cuidados intensivos, tras un lapso de 48 horas se evidenció oftalmoplejía completa del ojo contralateral izquierdo previamente sano asociado con pupila midriática no reactiva, considerando por clínica TSC izquierdo (figura 3).

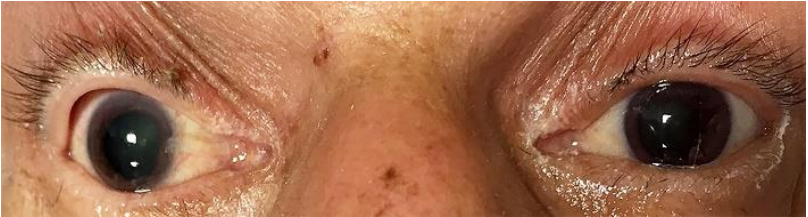

Figura 3. Pupila midriática no reactiva bilateral.

Durante su evolución, el paciente presentó como secuela compromiso ocular por las lesiones descritas a nivel de los nervios craneanos encargados de la movilidad ocular.

\section{DISCUSIÓN}

La trombosis bilateral del seno cavernoso como complicación de la enfermedad periodontal es un cuadro de presentación inusual. Los senos cavernosos contienen en su interior estructuras neurovasculares como la porción intracavernosa C4 de la arteria carótida interna, nervio oculomotor (III par), troclear (IV par), rama oftálmica y maxilar del trigémino ( $V$ par) y abducens ( $V I$ par) $)^{9}$. Reciben la mayor parte del retorno venoso de la cara a través de las venas oftálmicas y drenan en la vena yugular interna por medio de los senos petrosos superior e inferior; sin embargo, la ausencia de válvulas en sus conexiones vasculares predispone a infecciones de origen facial y senos paranasales ${ }^{10}$ como la descrita.

Como se evidenció en el caso clínico, el paciente con historia de diabetes mellitus mal controlada fue sometido en días previos al ingreso hospitalario a un procedimiento periodontal en dos órganos dentales localizados en el maxilar superior derecho. Este sitio posiblemente sirvió como puerta de entrada a un microorganismo infeccioso que desencadenó en principio una sinusitis maxilar derecha, seguida de celulitis orbitaria derecha y, en un lapso corto, se desarrolló como complicación una TSC ipsilateral (figura 4).

Esta hipótesis se fundamenta en el hecho de que la enfermedad periodontal asociada con diabetes mellitus con mal control metabólico aumenta el riesgo de complicaciones sistémicas ${ }^{11}$. Una periodontitis crónica puede resultar en un pobre control metabólico y, por otra parte, una diabetes mellitus fuera de metas, además de las reconocidas complicaciones micro y macrovasculares, puede 
derivar en una periodontitis; es decir, existe una relación bidireccional ${ }^{8}$.

Las complicaciones orales de la diabetes mellitus incluyen la caries dental, la xerostomía y las enfermedades periodontales, siendo estas últimas las más frecuentes y las que característicamente tienen un mayor riesgo de infección ${ }^{12}$. En un estudio tailandés, por ejemplo, se encontró una prevalencia de enfermedad periodontal del $73,5 \%$ en pacientes con diabetes mellitus tipo 2 , una correlación que fue significativa en aquellas personas con $\mathrm{HbA} 1 \mathrm{C} \geq 8,0 \%{ }^{11}$, dato que podría asociarse con el caso descrito.

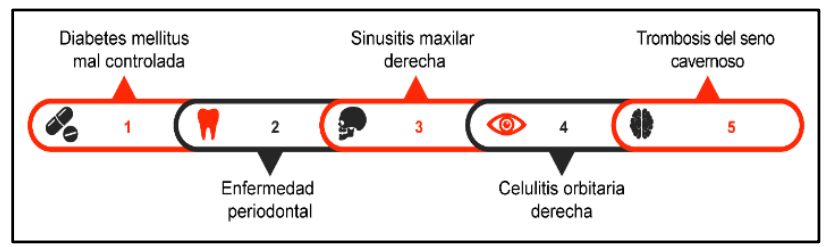

Figura 4. Secuencia de eventos clínicos en el paciente.

Los agentes etiológicos comúnmente asociados con TSC son las bacterias gram positivas, negativas y anaerobios, seguidas de los virus y hongos ${ }^{6}$. En el paciente se indicó tempranamente cubrimiento antibiótico empírico endovenoso, con mejoría inicial de la respuesta inflamatoria sistémica, razón por la cual se asoció como agente causal un germen posiblemente bacteriano. Esta hipótesis se reforzó tanto por la rápida instauración de la patología propia de las bacterias y el hallazgo de sinusitis maxilar derecha como por la complicación infecciosa por contigüidad de la enfermedad periodontal.

Los mecanismos implicados en la TSC séptica aún no están del todo claros. Sin embargo, se habla de una forma directa cuando la infección provoca por sí misma la trombosis, o indirecta cuando el proceso infeccioso precipita la trombosis en pacientes con factores de riesgo preexistentes ${ }^{13}$. En este caso, si bien el mecanismo pudo haber sido directo, no se puede descartar que la edad del paciente, entendida como tiempo de exposición a los factores de riesgo, y sus comorbilidades, especialmente la diabetes mellitus mal controlada, hayan condicionado un estado de hipercoagulabilidad e inmunosupresión, precipitando las manifestaciones trombóticas y el desenlace neurológico final ${ }^{12}$.

En un estudio publicado en el año 2018 se demostró cómo aquellos pacientes con diabetes mellitus con un pobre control metabólico ( $\mathrm{HbA} 1 \mathrm{c} \geq 11 \%)$ tienen mayor riesgo de desarrollar eventos infecciosos serios y de mortalidad $^{14}$. Asimismo, está demostrado que el efecto acumulativo de la senescencia inmune relacionada con la edad avanzada predispone a un mayor riesgo de infecciones por la diabetes mellitus de base que puede conducir a infecciones graves $y$ potencialmente mortales ${ }^{15}$.

La forma de presentación de la TSC es típicamente aguda, precedida de un cuadro de infección facial y/o de senos paranasales. Se desarrolla en un tiempo aproximado de una semana, aunque en pacientes con algún grado de inmunosupresión el curso de la enfermedad puede ser más rápido de lo habitual, pudiendo llegar a tener afectación bilateral entre las 24 y 48 horas, como se observó en el desenlace desfavorable y repentino del paciente ${ }^{1}$. Las manifestaciones clínicas más frecuentes son fiebre, ptosis palpebral secundario a la afectación del par craneal III, oftalmoplejía por lesión en los pares craneanos III, IV y VI, pérdida del reflejo corneano por alteración en la rama oftálmica del trigémino, quemosis, midriasis y edema periorbitario ${ }^{16}$, hallazgos que se dieron en el caso descrito.

La resonancia magnética cerebral constituye el estudio con mayor sensibilidad y especificidad para el diagnóstico. De esta forma se pueden detectar como signos de trombosis defectos de llenado en el seno cavernoso, junto con aumento de sus dimensiones o abombamiento de sus márgenes laterales ${ }^{7}$, imágenes que fueron visualizadas en el paciente mencionado.

La instauración de un tratamiento precoz es fundamental pues impacta directamente en la morbimortalidad. El pilar del manejo es la terapia antibiótica, cuya elección debe ser guiada de acuerdo con la sospecha de los gérmenes causales según los factores de riesgo e historia clínica individual, y el inicio temprano de anticoagulación 
que se asocia a mejores tasas de recanalización y menores déficit neurológico y mortalidad ${ }^{17,18}$.

\section{CONCLUSIÓN}

La TSC bilateral es una presentación inusual que debe considerarse dentro de los diagnósticos diferenciales en pacientes adultos mayores con algún grado de inmunosupresión, que cursen con infección a nivel de cabeza y cuello que rápidamente evolucione a un compromiso neurológico, especialmente a nivel orbitario, ya que un diagnóstico oportuno impactará en los desenlaces. Por otra parte, cabe resaltar la importancia de realizar una previa verificación del control de las patologías de base de un paciente que se somete a cualquier procedimiento odontológico ya que, como en este caso, una diabetes mellitus mal controlada predispone a infecciones locales y a distancia que podrían ser prevenibles con un manejo interdisciplinario.

\section{DECLARACIÓN SOBRE CONFLICTOS DE INTERESES}

Los autores declaran que no existe conflicto de intereses.

\section{CONTRIBUCIÓN DE LOS AUTORES}

Primer autor: trabajo clínico, búsqueda bibliográfica y redacción del manuscrito.

Segundo autor: trabajo clínico, búsqueda bibliográfica y redacción del manuscrito.

Tercer autor: diseño metodológico, redacción y ajuste del manuscrito.

Cuarto autor: trabajo clínico y redacción del manuscrito.

Quinto autor: trabajo clínico y redacción del manuscrito.

\section{REFERENCIAS BIBLIOGRÁFICAS}

1. Dinkin M, Patsalides A, Ertel M. Diagnosis and Management of Cerebral Venous Diseases in NeuroOphthalmology: Ongoing Controversies. Asia-Pacific Journal of Ophthalmology. 2019; 8(1): 73-85. Doi: http://dx.doi.org/10.22608/APO.2018239
2. Cho JY, Kim HM, Ryu JY. Cavernous sinus thrombosis progression from trismus. Journal of the Korean Association Oral and Maxillofacial Surgeons. 2015; 41(1): 43-7. Doi: http://dx.doi.org/10.5125/jkaoms.2015.41.1.43

3. Guenther G, Arauz A. Trombosis venosa cerebral: aspectos actuales del diagnóstico y tratamiento. Neurología. 2011; 26(8): 488-98. Doi: http://dx.doi.org/10.1016/j.nrl.2010.09.013

4. Khatri IA, Wasay M. Septic cerebral venous sinus thrombosis. Journal of the Neurological Sciences. 2016; 362: 221-7. Doi: http://dx.doi.org/10.1016/j.jns.2016.01.035

5. Desa V, Green R. Cavernous Sinus Thrombosis: Current Therapy. Journal of Oral and Maxillofacial Surgery. 2012; 70(9): 2085-91. Doi: http://dx.doi.org/10.1016/j.joms.2011.09.048

6. Hsu CW, Tsai WC, Lien CY, Lee JJ, Chang WN. The clinical characteristics, implicated pathogens and therapeutic outcomes of culture-proven septic cavernous sinus thrombosis. Journal of Clinical Neuroscience. 2019; 68: 111-6. Doi: http://dx.doi.org/10.1016/j.jocn.2019.07.022

7. Bhatia $H$, Kaur R, Bedi R. MR imaging of cavernous sinus thrombosis. European Journal of Radiology Open. 2020; (7): $100226 . \quad$ Doi: http://dx.doi.org/10.1016/j.ejro.2020.100226

8. Bui FQ, Coutinho CL, Huynh B, Trinh A, Liu J, Woodward J, et al. Association between periodontal pathogens and systemic disease. Biomedical Journal. 2018; 42(1): 27-35. Doi: http://dx.doi.org/10.1016/j.bj.2018.12.001

9. Mahalingam HV, Mani S, Patel B, Prabhu K, Alexander M, Fatterpekar G, et al. Imaging Spectrum of Cavernous Sinus Lesions with Histopathologic Correlation. Radiographics. 2019; 39(3): 795-819. Doi: http://dx.doi.org/10.1148/rg.2019180122

10. Gallagher JP. Septic thrombosis of the cavernous sinus. The Medical Annals of the District of Columbia. 1960; 29: 278-83. Available at: https://pubmed.ncbi.nlm.nih.gov/13826082/ 
11. Hsu YJ, Lin KD, Chen JH, Lee MY, Lin YC, Yen FC, et al. Periodontal Treatment Experience Associated with Oral Health-Related Quality of Life in Patients with Poor Glycemic Control in Type 2 Diabetes: A Case-Control Study. International Journal of Environmental Research ans Public Health. 2019; 16(20): $4011 . \quad$ Doi: http://dx.doi.org/10.3390/ijerph16204011

12. Rohani B. Oral manifestation in patients with diabetes mellitus. World Journal of Diabetes. 2019; 10(9): 485-9.

Doi:

http://dx.doi.org/10.4239/wjd.v10.i9.485

13. Colbert S, Cameron M, Williams J. Septic thrombosis of the cavernous sinus and dental infection. British Journal of Oral and Maxillofacial Surgery. 2011; 49(6): e25-e26. Doi: http://dx.doi.org/10.1016/j.bjoms.2010.07.004

14. Critchley JA, Carey IN, Harris T, Dewilde S, Hosking D, Cook D, et al. Glycemic Control and Risk of Infections Among People with Type 1 or Type 2 Diabetes in a Large Primary Care Cohort Study. Diabetes Care. 2018; 41(10): 2127-35. Doi: http://dx.doi.org/10.2337/dc18-0287

15. Rajagopalan S. Serious Infections in Elderly Patients with Diabetes Mellitus. Clinical Infectious Diseases. 2005; 40(7): 990-6. Doi: http://dx.doi.org/10.1086/427690

16. Bhatia K, Jones NS. Septic cavernous sinus thrombosis secondary to sinusitis: are anticoagulants indicated? A review of the literature. The Journal of Laryngology and Otology. 2017; 116(9): $\quad$ 667-76. Doi: http://dx.doi.org/10.1258/002221502760237920

17. Xu W, Gao L, Li T, Shao A, Zhang J. Efficacy and risks of anticoagulation for cerebral venous thrombosis. Medicine Baltimore. 2018; 97(20): e10506.

Doi:

http://dx.doi.org/10.1097/MD.0000000000010506

18. Coutinho J, de Bruijn SF, Deveber G, Stam J. Anticoagulation for cerebral venous sinus thrombosis. The Cochrane Database of Systematic Reviews. 2011; 2011(8): CD002005. Doi: http://dx.doi.org/10.1002/14651858.cd002005.pub 2 